Original Article

\title{
Comprehensive geriatric assessment of effects of hospitalization and long-term rehabilitation of patients following lower extremity arthroplasty
}

Yuma Sonoda, PhD, $\mathrm{OT}^{1)^{*}}$, Shinichiro Sawano, $\mathrm{PT}^{1)}$, Yuka Kojima, $\mathrm{PT}^{1)}$, Masato Kugo, $\mathrm{PT}^{1)}$, Masashi Taniguchi, PT, MSc ${ }^{1)}$, Shoji Maegawa, $\mathrm{PT}^{1)}$, Taku Kawasaki, PhD, $\mathrm{MD}^{1,2)}$

1) Rehabilitation Units, Shiga University of Medical Science: Seta Tsukinowa-cho, Otsu, Shiga 5202192, Japan

2) Department of Orthopedic Surgery, Shiga University of Medical Science, Japan

\begin{abstract}
Purpose] This study was performed to examine the effects of subacute physical therapy (PT) on activities of daily living (ADL), quality of life, and geriatric aspects of patients who underwent total knee arthroplasty (TKA) or total hip arthroplasty (THA). [Subjects] The subjects were TKA $(n=56)$ and THA $(n=39)$ patients who received PT on the first day of independent ADL (up to 2 weeks) and just prior to discharge (4 weeks). [Methods] The functional independence measure (FIM), grip strength, knee extension strength (KES), timed up and go (TUG) test, mini-mental state examination (MMSE), geriatric depression scale short form (GDS-15), fall efficacy scale (FES), and medical outcome study 8 -item short-form health survey (SF-8) were used as outcome measure, and comorbidity involvement was also investigated. [Results] Improvements in FIM, KES, TUG, GDS-15, FES, and SF-8 scores were seen in both groups (effect size, 0.31-0.87). Poor PT effects were found for THA patients aged $\geq 65$ years, for TKA and THA patients with an MMSE score $\leq 28$, and for THA patients with two or more comorbidities. [Conclusion] Positive effects were seen in patients who received PT at 2-4 weeks after surgery. Thus, additional PT for approximately 2 weeks after the beginning of independent ADL may be beneficial.

Key words: Total knee arthroplasty (TKA), Total hip arthroplasty (THA), Comprehensive geriatric assessment (CGA)
\end{abstract}

(This article was submitted Nov. 24, 2015, and was accepted Dec. 23, 2015)

\section{INTRODUCTION}

Total knee arthroplasty (TKA) and total hip arthroplasty (THA) are the most commonly performed surgical procedures for the lower limbs with orthopedic disease. The aims of surgery are to alleviate pain, and improve activities of daily living (ADL) and physical activity ${ }^{1-3}$. The purpose of post-operative rehabilitation for such patients is to improve physical function, ADL, and quality of life (QOL), as well as extend healthy life expectancy ${ }^{2-5)}$. However, hospital stays and the time of rehabilitation following lower limb arthroplasty have recently been reduced in light of increased medical expenses in Western countries, which is different from the situation in Japan ${ }^{6,7)}$. The Uniform Data System for Medical Rehabilitation (UDSMR) Functional Independence Measure (FIM) system (FIM ${ }^{\circledR}$ instrument) is the largest case registration database used in the field of rehabilitation in the United States, and Dr. Granger is one of the developers of the system. Using the UDSMR-FIM, Granger et al. ${ }^{7)}$ reported that the FIM score at discharge of patients who underwent TKA and THA was $108.4 \pm 11.0$ points in measurements conducted in 2000 compared to $101.7 \pm 12.9$ points in 2008 , showing a tendency for discharge with slightly poorer ADL, while the average length of hospital stay in 2008 was found to be $9.0 \pm 4.1$ days.

Although the numbers of TKA and THA procedures continue to increase each year, and shortened hospital stay is being promoted, hospitalization of about 3-4 weeks remains common in Japan. For patients in Western countries who undergo TKA

\footnotetext{
*Corresponding author. Yuma Sonoda (E-mail: yuma@belle.shiga-med.ac.jp)

(C)2016 The Society of Physical Therapy Science. Published by IPEC Inc.

This is an open-access article distributed under the terms of the Creative Commons Attribution Non-Commercial No Derivatives (by-nc-nd) License $<$ http://creativecommons.org/licenses/by-nc-nd/4.0/>.
} 


\begin{tabular}{|c|c|c|c|c|c|c|c|c|}
\hline \multirow{2}{*}{$\begin{array}{l}\text { Type of arthroplasty } \\
\text { Week }\end{array}$} & \multicolumn{4}{|c|}{ Total Knee } & \multicolumn{4}{|c|}{ Total Hip } \\
\hline & 1 & 2 & 3 & 4 & 1 & 2 & 3 & 4 \\
\hline \multicolumn{9}{|l|}{ Gait } \\
\hline \multicolumn{9}{|l|}{ Walker } \\
\hline Cane & & $\bullet$ & $\bullet$ & 0 & & 0 & $\bullet$ & 0 \\
\hline Free & & & & 0 & & & & 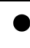 \\
\hline \multicolumn{9}{|l|}{ Exercise } \\
\hline ROM & $\bullet$ & $\bullet$ & $\bullet$ & 0 & $\bullet$ & $\bullet$ & $\bullet$ & $\bullet$ \\
\hline \multicolumn{9}{|l|}{ Knee flexion } \\
\hline Knee extension & $\bullet$ & $\bullet$ & $\bullet$ & 0 & - & $\bullet$ & $\bullet$ & $\bullet$ \\
\hline Hip extension & $\bullet$ & - & - & - & - & $\bullet$ & - & $\bullet$ \\
\hline Hip abduction & $\bullet$ & - & - & 0 & & & $\bullet$ & $\bullet$ \\
\hline \multicolumn{9}{|l|}{ ADL } \\
\hline Standing & - & - & - & 0 & - & 0 & 0 & 0 \\
\hline Stairs & & & - & $\bullet$ & & - & - & $\bullet$ \\
\hline Bath/floor & & & $\bullet$ & 0 & & & 0 & 0 \\
\hline Evaluation & & - & & 0 & & 0 & & 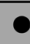 \\
\hline
\end{tabular}

Fig. 1. Physical therapy program following total knee arthroplasty or total hip arthroplasty Time line: week 1 (POD, post-operative day 1-7), week 2 (POD 8-14), week 3 (POD 15-21), week 4 (POD 22-28).

Additionally, ankle pumps and walker-gait devices were used to prevent deep vein thrombosis between POD 1 and hospital discharge.

or THA, rehabilitation strategies with high cost-effectiveness in the light of the risk of adverse events for elderly patients have been developed ${ }^{8)}$, with long-term clinical results such as physical function and QOL reported ${ }^{2,9)}$. For rehabilitation during the recovery period after the sub-acute stage following TKA and THA, Western patients are generally discharged to home. However, in consideration of the aging population in Japan, it is important to evaluate the usefulness of rehabilitation during the very early stage, and that performed for a short period, in addition to both physical function and mental health, by determining the overall effects, including geriatric aspects.

Elderly patients often have complications of multiple diseases or decreased ADL prior to surgery; thus they, require comprehensive medical assistance. As for an effective methodology, it is important to not only perform medical evaluations but also comprehensive assessments of three aspects of life function, mental, social, and environmental aspects, in order to understand related problems and increase in QOL, in other words, the comprehensive geriatric assessment (CGA). In previous studies that utilized CGA, the effects of mortality decline, reduced length of hospitalization, extended stay at home, reduced admission rate, maintenance of bodily functions, fall prevention, preservation of cognitive function, management of perioperative delirium, reduced medical costs, and coordinated hyper-medication have been reported ${ }^{10-12)}$.

The present study was performed to verify the usefulness and validity of discharge time after four weeks of rehabilitation for patients who underwent a TKA or THA procedure. Changes in motor function, cognitive function, mood, and QOL were assessed at the time of independent ADL and at discharge. From those findings, the significance of convalescence for rehabilitation of lower limb prosthetic joints was investigated by taking into account the impact of geriatric and comorbid conditions in line with the concept of the CGA.

\section{SUBJECTS AND METHODS}

A total of 111 patients (mean age 69.7 \pm 8.7 years; TKA, $n=68$; THA, $n=43$ ) underwent a TKA or THA procedure at the Department of Orthopedic Surgery at Shiga University of Medical Science Hospital between June 2013 and April 2014. Each patient began physical therapy (PT) from the day after surgery, and were transferred to the convalescence rehabilitation ward (CRW) from the orthopedic ward and underwent re-evaluation. Criteria for admission to the study were C-reactive protein $\leq 3.0 \mathrm{mg} / \mathrm{dl}$, independent gait with an assistive device, and commencement of stair training. Exclusion criteria were presence of osteonecrosis or rheumatoid arthritis before surgery, neurological symptoms such as stroke or Parkinson's disease, advanced disuse syndrome, and dementia [mini-mental state examination (MMSE) score $\leq 23$ ]. Some patients underwent TKA with a lateral parapatellar approach, and some patients underwent THA with Dall's approach. The pre-operative characteristics of the subjects are shown in Table 1. In addition, the critical paths of the PT program at our hospital for TKA and THA patients are presented in Fig. 1. This study was performed in accordance with the Declaration of Helsinki. The protocol was approved by the medical ethics committees of our university and all participants provided their written informed consent 
Table 1. Characteristics of patients who underwent total knee arthroplasty or total hip arthroplasty

\begin{tabular}{|c|c|c|}
\hline & Total knee $(\mathrm{n}=56)$ & Total hip $(n=39)$ \\
\hline & mean $(\mathrm{SD})$ & mean $(\mathrm{SD})$ \\
\hline Length of stay, days & $29.8(5.2)$ & $30.8(8.0)$ \\
\hline Onset (from surgery to CRW) & $13.9(4.2)$ & $13.4(3.9)$ \\
\hline CRW & $15.8(4.6)$ & $17.4(8.1)$ \\
\hline Gender, female & $46(82.1 \%)$ & $35(89.7 \%)$ \\
\hline Age, years & $71.6(8.3)$ & $67.7(9.0)$ \\
\hline$<65$ & $13(23.2 \%)$ & $16(41.0 \%)$ \\
\hline$\geq 65$ & $43(76.8 \%)$ & $23(59.0 \%)$ \\
\hline Height, cm & $152.3(7.3)$ & $152.1(6.1)$ \\
\hline Weight, kg & $60.4(10.9)$ & $54.4(10.4)$ \\
\hline BMI, $\mathrm{kg} / \mathrm{m}^{2}$ & $25.9(3.7)$ & $23.4(3.7)$ \\
\hline KSS or mHHS score ${ }^{\S}$ & $71.2(25.2)$ & $54.9(11.7)$ \\
\hline \multirow[t]{2}{*}{ Surgical approach } & medial parapatellar: $32(57.1 \%)$ & antero-lateral: $39(100 \%)$ \\
\hline & midvastus: 24 (42.9\%) & \\
\hline Surgical side, right & $31(55.4 \%)$ & $21(53.8 \%)$ \\
\hline Dominant leg, right & $52(92.9 \%)$ & $37(94.9 \%)$ \\
\hline Dominant hand, right & $56(100 \%)$ & $38(97.4 \%)$ \\
\hline Total FIM score, performance (adm.) & $111.5(5.2)$ & $108.5(6.7)$ \\
\hline Total MMSE score & 28.3 (1.9); median, 29 & 28.3 (2.8); median, 29 \\
\hline \multicolumn{3}{|l|}{ Non-musculoskeletal comorbidities } \\
\hline Hypertension & $36(64.3 \%)$ & $20(51.3 \%)$ \\
\hline Chronic heart failure & $19(33.9 \%)$ & $5(12.8 \%)$ \\
\hline Diabetes mellitus & $12(21.4 \%)$ & $2(5.1 \%)$ \\
\hline Hyperlipidemia & $24(42.9 \%)$ & $15(38.5 \%)$ \\
\hline Asthma or COPD & $5(8.9 \%)$ & $2(5.1 \%)$ \\
\hline \multicolumn{3}{|l|}{ Sensory impairments } \\
\hline Vison impairment & $7(12.5 \%)$ & $4(10.3 \%)$ \\
\hline Hearing impairment & $5(8.9 \%)$ & $2(5.1 \%)$ \\
\hline \multicolumn{3}{|l|}{ Number of comorbidities } \\
\hline 0 & $8(14.3 \%)$ & $13(33.3 \%)$ \\
\hline 1 or 2 & $33(58.9 \%)$ & $20(51.3 \%)$ \\
\hline$\geq 3$ & $15(26.8 \%)$ & $6(15.4)$ \\
\hline Number of falls & $2(35.7 \%)^{\oplus}$ & $1(25.6 \%)^{\llbracket}$ \\
\hline Discharge setting, home & $55(98.2 \%)$ & $39(100 \%)$ \\
\hline Living situation, alone or with others & $8(14.3 \%)$ or $48(85.7 \%)$ & $5(12.8 \%)$ or $34(87.2 \%)$ \\
\hline
\end{tabular}

CRW: convalescence rehabilitation ward; BMI: body mass index; FIM: functional independence measure (at admission); MMSE: mini-mental state examination; SD: standard deviation

\$Pre-operative score; total knee society score (KSS) for TKA, and total modified Harris hip score (mHHS) for THA.

TPermil (\%); number of falls/total inpatient number $\times 1,000=$ sum during study period

before enrollment.

The subjects were examined at the time of admission to the study (transfer from orthopedic department to rehabilitation unit) and at the time of hospital discharge. The main outcome measure was the FIM, which has 18 items covering 6 domains (self-care, sphincter control, transfer, locomotion, communication, social cognition). Each item is rated on a scale from 1 (complete dependence) to 7 (complete independence), with higher scores representing greater functional independence (range 18 to 126) in ADL. In addition, grip and knee extensor strength were measured, and the timed up and go test (TUG) conducted to assess motor function. Knee extensor strength was assessed using an IsoForce GT-360 device (OG Giken Co Ltd, Okayama, Japan) while the subjects performed isometric contraction for 3 seconds. Torque was calculated by multiplying the strength of the lever arm (distance between position of force sensor and knee extensor strength) and expressed as a percentage of body weight $(\mathrm{N} \cdot \mathrm{m} / \mathrm{kg})$. The TUG test measures the time (in seconds) required by a subject to stand up from an armless chair (chair seat height up to $40 \mathrm{~cm}$ ), walk a distance of 3 meters, then turn, walk back to the chair, and sit down. 
Table 2. Results of outcome measures before and after surgery for patients who underwent total knee arthroplasty or total hip arthroplasty

\begin{tabular}{|c|c|c|c|c|c|c|}
\hline & \multicolumn{2}{|c|}{ Total knee $(n=56)$} & \multirow{3}{*}{ ES } & \multicolumn{2}{|c|}{ Total hip $(\mathrm{n}=39)$} & \multirow{3}{*}{ ES } \\
\hline & \multicolumn{2}{|l|}{ mean $(\mathrm{SD})$} & & \multicolumn{2}{|c|}{ mean $(\mathrm{SD})$} & \\
\hline & Admission & Discharge & & Admission & Discharge & \\
\hline Total FIM score, capability & $113.9(5.5)$ & $121.7(3.8)^{*}$ & 0.86 & $111.9(6.5)$ & $119.9(5.5)^{*}$ & 0.87 \\
\hline transfer, tub & $4.5(2.0)$ & $6.1(0.5)^{*}$ & 0.72 & $4.8(1.7)$ & $5.9(0.6)^{*}$ & 0.69 \\
\hline walk & $6.1(0.8)$ & $6.5(0.5)^{*}$ & 0.58 & $6.1(0.2)$ & $6.3(0.5)^{*}$ & 0.46 \\
\hline stairs & $3.0(2.3)$ & $6.0(0.3)^{*}$ & 0.78 & $3.0(2.2)$ & $5.8(0.6)^{*}$ & 0.81 \\
\hline Grip, right (kg) & $20.9(7.8)$ & $20.7(7.9)$ & & $21.2(7.3)$ & $21.4(7.1)$ & \\
\hline Grip, left (kg) & $19.8(7.4)$ & $20.1(7.9)$ & & $20.5(6.8)$ & $20.8(6.9)$ & \\
\hline Knee extension, right $(\mathrm{Nm} / \mathrm{kg})$ & $0.8(0.5)$ & $1.0(0.4)^{*}$ & 0.74 & $1.0(0.4)$ & $1.1(0.4)^{*}$ & 0.52 \\
\hline Knee extension, left (Nm/kg) & $0.8(0.4)$ & $0.9(0.4)^{*}$ & 0.69 & $1.0(0.4)$ & $1.1(0.4)^{*}$ & 0.46 \\
\hline TUG $(\mathrm{sec})$ & $13.7(5.7)$ & $10.0(2.9)^{*}$ & 0.84 & $15.5(6.0)$ & $11.5(4.7)^{*}$ & 0.8 \\
\hline Total MMSE score & $28.3(1.9)$ & $28.1(2.1)$ & & $28.3(2.8)$ & $28.2(2.5)$ & \\
\hline Total GDS-15 score & $4.2(3.2)$ & $2.6(2.5)^{*}$ & 0.55 & $3.6(3.0)$ & $3.1(3.2)^{*}$ & 0.34 \\
\hline \multicolumn{7}{|l|}{ SF-8 score } \\
\hline physical function & $35.2(10.3)$ & $42.4(5.5)^{*}$ & 0.56 & $39.0(10.0)$ & $44.0(5.2)^{*}$ & 0.44 \\
\hline role physical & $33.7(11.1)$ & $42.0(7.5)^{*}$ & 0.57 & $38.9(9.0)$ & $45.0(6.1)^{*}$ & 0.5 \\
\hline bodily pain & $36.1(5.5)$ & $41.2(6.6)^{*}$ & 0.57 & $39.4(7.4)$ & $45.3(7.8)^{*}$ & 0.51 \\
\hline general health & $47.4(7.4)$ & $51.6(6.5)^{*}$ & 0.45 & $48.3(7.5)$ & $52.2(5.9)^{*}$ & 0.43 \\
\hline vitality & $46.0(7.5)$ & $49.9(6.1)^{*}$ & 0.49 & $48.9(7.1)$ & $51.3(5.7)^{*}$ & 0.35 \\
\hline social function & $40.0(8.8)$ & $46.7(8.1)^{*}$ & 0.56 & $42.3(9.9)$ & $42.8(9.5)$ & \\
\hline role emotional & $39.1(13.3)$ & $45.8(7.5)^{*}$ & 0.43 & $42.3(10.8)$ & $47.0(6.3)$ & \\
\hline mental health & $46.8(6.4)$ & $50.0(5.5)^{*}$ & 0.36 & $48.4(6.7)$ & $50.8(6.0)^{*}$ & 0.34 \\
\hline PCS & $33.7(7.6)$ & $40.8(5.6)^{*}$ & 0.74 & $7.8(8.4)$ & $43.8(5.3)^{*}$ & 0.57 \\
\hline MCS & $47.3(9.4)$ & $50.8(6.6)^{*}$ & 0.37 & $48.6(7.3)$ & $49.5(5.7)$ & \\
\hline Total FES score & $43.6(29.7)$ & $52.4(28.9)^{*}$ & 0.31 & $48.5(27.6)$ & $60.4(34.0)^{*}$ & 0.36 \\
\hline
\end{tabular}

${ }^{*} \mathrm{p}<0.05$ : a significant difference, Wilcoxon's signed-rank test. SD: standard deviation; ES: effect size; $r=Z$-score $/ \sqrt{ }(N)$, FES: fall efficacy scale; FIM: functional independence measure; GDS-15: geriatric depression scale short form (15 items); MCS: mental component summary; MMSE: mini-mental state examination; PCS: physical component summary; SF-8: 8-item short-form health survey; TUG: timed up and go test

In this study, it was used to assess ambulatory ability. Cognitive function was assessed with using the MMSE (score range, 0 to 30; lower scores indicate worse performance). Depression mood was assessed using the geriatric depression scale short form (GDS-15; score range, 0 to 15; higher scores indicate worse performance). Health-related QOL (HRQOL) was assessed using the MOS 8-item short-form health survey (SF-8; norm-based score, 50; lower scores indicate worse performance). In order to evaluate the fear of falling and self-efficacy, the fall efficacy scale (FES) developed by Takenaka et al. ${ }^{13)}$ was used to examine 15 activities associated with ADL. The FES rates self-confidence in avoiding falls on a 10-point scale (1: absolutely no confidence, 10: extremely confident), and higher scores show strong confidence of avoiding falls. In the present study, the value of 0 was added to represent "do not know" or "have never been" to the possible answers.

Additionally, gender, age, height, weight, dominant hand ${ }^{14)}$ and leg ${ }^{15)}$, presence or absence of a comorbidity such as hypertension, chronic heart failure due to ischemic heart disease or others, diabetes mellitus, hyperlipidemia, respiratory disease (chronic obstructive pulmonary disease, asthma), visual impairment (waiting for cataract surgery or glaucoma during treatment), hearing impairment (hypacusia), discharge destination, family structure, and occurrence of a fall during hospitalization were all investigated.

For the assessment of physical function, the better score of 2 trials was used. Statistical analysis was performed for all of the data of the TKA and THA patients using non-parametric tests. The Wilcoxon signed-rank test was used to examine differences between admission and discharge, and the size of the effect was calculated. The Mann-Whitney U test was used to examine the relationships among geriatric factors, such as age, MMSE, number of comorbidities, and surgical side. For this purpose, the patients were divided into two age groups, with 65 years as the dividing point. The CRW is an intermediary facility used prior to discharge to home, and some inpatients after discharge from the hospital are certified for long-term nursing-care insurance according to need. The present subjects were divided into high-scoring (High) and low-scoring (Low) groups based on the MMSE median. All statistical analyses were performed using SPSS for Windows (version 22.0, IBM, Tokyo, Japan) and values of $\mathrm{p}<0.05$ were considered to indicate statistical significance. 
Table 3. Differences between admission and discharge based on age

\begin{tabular}{lcccc}
\hline \multirow{2}{*}{$\Delta)=$ Discharge - Admission } & \multicolumn{2}{c}{ Total knee, mean $(\mathrm{SD})$} & \multicolumn{2}{c}{ Total hip, mean (SD) } \\
\cline { 2 - 4 } Age in years $^{\dagger}$ & $<65$ & $\geq 65$ & $<65$ & $\geq 65$ \\
& $(\mathrm{n}=13)$ & $(\mathrm{n}=43)$ & $(\mathrm{n}=16)$ & $(\mathrm{n}=23)$ \\
\hline Total FIM score, capability & $8.2(4.5)$ & $7.6(4.4)$ & $8.0(2.9)$ & $8.0(4.2)$ \\
FIM efficiency & $0.6(0.4)$ & $0.5(0.3)$ & $0.5(0.2)$ & $0.5(0.3)$ \\
Grip, right $(\mathrm{kg})$ & $-0.3(2.3)$ & $-0.1(2.6)$ & $-0.1(2.7)$ & $0.4(2.4)$ \\
Grip, left $(\mathrm{kg})$ & $0.9(2.6)$ & $0.1(2.1)$ & $0.4(2.7)$ & $0.2(2.4)$ \\
Knee extension, right $(\mathrm{Nm} / \mathrm{kg})$ & $0.2(0.2)$ & $0.2(0.2)$ & $0.1(0.2)$ & $0.1(0.2)$ \\
Knee extension, left $(\mathrm{Nm} / \mathrm{kg})$ & $0.2(0.2)$ & $0.1(0.2)$ & $0.0(0.2)$ & $0.2(0.2)$ \\
TUG (sec) & $-4.1(3.4)$ & $-3.6(4.1)$ & $-2.9(2.7)$ & $-4.7(4.8)$ \\
Total MMSE score & $0.2(1.3)$ & $-0.2(1.7)$ & $-0.1(1.1)$ & $-0.1(1.8)$ \\
Total GDS-15 score & $-2.5(2.3)$ & $-1.2(2.4)$ & $-0.9(1.8)$ & $-0.3(2.4)$ \\
SF-8 score & & & & $3.7(9.7)$ \\
physical function & $8.3(10.0)$ & $7.0(10.7)$ & $6.7(12.2)$ & $4.3(11.0)$ \\
role physical & $7.9(11.1)$ & $8.5(12.2)$ & $8.6(10.8)$ & $3.1(9.2)^{*}$ \\
bodily pain & $7.0(9.4)$ & $4.6(6.0)$ & $9.9(7.5)$ & $2.9(7.3)$ \\
general health & $4.2(8.0)$ & $4.2(8.3)$ & $5.3(8.2)$ & $1.5(7.2)$ \\
vitality & $4.4(5.7)$ & $3.8(7.3)$ & $3.9(6.7)$ & $-0.5(10.0)$ \\
social function & $5.5(7.0)$ & $7.1(10.1)$ & $1.9(6.2)$ & $3.6(10.5)$ \\
role emotional & $9.1(15.2)$ & $6.0(14.1)$ & $6.2(12.7)$ & $2.1(7.1)$ \\
mental health & $3.2(6.3)$ & $3.2(7.2)$ & $2.8(4.7)$ & $3.8(10.4)$ \\
PCS & $7.6(7.4)$ & $6.9(7.2)$ & $9.1(7.7)$ & $0.9(7.1)$ \\
MCS & $3.8(8.1)$ & $3.4(10.2)$ & $1.0(6.3)$ & $2.1(46.3)^{*}$ \\
Total FES score & $5.9(43.7)$ & $9.7(28.5)$ & $26.0(33.4)$ &
\end{tabular}

†The groups were divided based on age, with 65 years old as the cut-off.

${ }_{*}<$ 0.05: a significant difference, Mann-Whitney's U test. SD: standard deviation; FES: fall efficacy scale; FIM: functional independence measure; GDS-15: geriatric depression scale short form (15 items); MCS: mental component summary; MMSE: mini-mental state examination; PCS: physical component summary; SF-8: 8-item short-form health survey; TUG: timed up and go test

\section{RESULTS}

In accordance with the inclusion criteria, patients who were followed after hospital discharge were subjected to analysis. The TKA group consisted of 56 patients (46 females, 10 males), with a mean age of $71.6 \pm 8.3$ years (range 52-86 years) and mean body mass index (BMI) of $25.9 \pm 3.7 \mathrm{~kg} / \mathrm{m}^{2}\left(19.4-35.0 \mathrm{~kg} / \mathrm{m}^{2}\right)$, and the THA group consisted of 39 patients $(35$ femlaes, 4 males), with a mean age of $67.7 \pm 9.0$ years (49-81 years) and mean body mass index (BMI) of $23.4 \pm 3.7 \mathrm{~kg} / \mathrm{m}^{2}$ $\left(17.5-33.7 \mathrm{~kg} / \mathrm{m}^{2}\right)$. As for the operative procedures, in the TKA group, 24 patients underwent a midvastus approach and 32 a medial parapatellar approach, while all patients in the THA group underwent an antero-lateral approach.

The evaluation period after entering the CRW for patients in both groups ranged from 2 to 4 weeks after surgery (Table 1). The duration of hospitalization was $29.8 \pm 5.2$ days in the TKA group and 30.8 \pm 8.0 days in the THA group. PT was conducted during CRW stays of 15.8 \pm 4.6 and 17.4 \pm 8.1 days for the TKA and THA groups, respectively. Furthermore, the two groups' initial values of FIM were 111.5 \pm 5.2 and 108.5 \pm 6.7 points, and of MMSE were $28.3 \pm 1.9$ and $28.3 \pm 2.8$ points (median 29 points for both groups), respectively. For the TKA and THA groups, surgery on the right side was performed for $55.4 \%$ and $53.8 \%$ of the subjects, respectively, and the comorbidity prevalences were $85.7 \%$ and $66.7 \%$, the falls rates were $3.6 \%$ and $2.6 \%$, and the home discharge rates were $98.2 \%$ and $100 \%$, respectively (Table 1). Hypertension and hyperlipidemia coexisted in most patients in both groups, while many also had musculoskeletal disease along with two or more diseases. Furthermore, scores for the Waterloo Footedness Questionnaire revealed right-leg dominance in 92.9\% of the TKA and $94.9 \%$ of the THA patients (Table 1). Regarding the knee extension strength of the TKA group, there was no significant difference between the medial parapatellar $(n=32)$ and midvastus $(n=24)$ approaches, or between the right $(p=0.246)$ and left $(p=0.141)$ sides at the time of admission to the CRW, or between the right $(p=0.96)$ and left $(p=0.371)$ sides at discharge.

There were significant differences between the groups with regard to functional improvements at 2 and 4 weeks after the surgery. In comparisons of the scores between admission and discharge in the TKA and THA groups, overall good clinical results were shown for FIM (ADL), motor function (knee extensor strength, TUG), mood (GDS and FES), and HRQOL (SF-8) (Table 2). The clinical effect was moderate to large in both groups, while the physical effects were more intense than 
Table 4. Differences in functions between dominant leg and surgery side

\begin{tabular}{lcccc}
\hline \multirow{2}{*}{$\Delta)$ Discharge - Admission } & \multicolumn{2}{c}{ Total knee, mean $(\mathrm{SD})$} & \multicolumn{2}{c}{ Total hip, mean (SD) } \\
\cline { 2 - 5 } Dominant leg \& surgery side & $\begin{array}{c}\text { Ipsilateral } \\
(\mathrm{n}=32)\end{array}$ & $\begin{array}{c}\text { Contra } \\
(\mathrm{n}=24)\end{array}$ & $\begin{array}{c}\text { Ipsilateral } \\
(\mathrm{n}=21)\end{array}$ & $\begin{array}{c}\text { Contra } \\
(\mathrm{n}=18)\end{array}$ \\
\hline Total FIM score, capability & $8.3(4.4)$ & $7.0(4.4)$ & $8.1(4.4)$ & $7.8(2.9)$ \\
FIM efficiency & $0.5(0.3)$ & $0.5(0.3)$ & $0.5(0.2)$ & $0.5(0.2)$ \\
Grip, right $(\mathrm{kg})$ & $-0.1(2.6)$ & $-0.3(2.5)$ & $0.6(2.4)$ & $-0.3(2.6)$ \\
Grip, left $(\mathrm{kg})$ & $0.1(2.3)$ & $0.5(2.2)$ & $0.6(2.5)$ & $-0.1(2.4)$ \\
Knee extension, right $(\mathrm{Nm} / \mathrm{kg})$ & $0.3(0.3)$ & $0.1(0.1)^{*}$ & $0.1(0.2)$ & $0.1(0.2)$ \\
Knee extension, left $(\mathrm{Nm} / \mathrm{kg})$ & $0.1(0.2)$ & $0.2(0.2)^{*}$ & $0.1(0.2)$ & $0.1(0.2)$ \\
TUG (sec) & $-4.3(4.1)$ & $-3.0(3.6)$ & $-3.1(3.6)$ & $-4.9(4.7)$ \\
Total MMSE score & $0.1(1.8)$ & $-0.4(1.4)$ & $-0.1(1.7)$ & $-0.1(1.3)$ \\
Total GDS-15 score & $-1.2(2.3)$ & $-2.0(2.6)$ & $-0.7(1.6)$ & $-0.4(2.7)$ \\
SF-8 score & & & & $5.9(12.2)$ \\
physical function & $8.2(11.2)$ & $6.1(9.5)$ & $4.1(9.6)$ & $5.8(10.7)$ \\
role physical & $7.9(11.7)$ & $8.9(12.3)$ & $6.4(11.5)$ & $4.3(10.2)$ \\
bodily pain & $5.4(6.2)$ & $4.8(8.0)$ & $7.2(8.1)$ & $2.4(6.2)$ \\
general health & $4.4(8.2)$ & $3.9(8.2)$ & $5.1(8.8)$ & $1.0(5.6)$ \\
vitality & $2.6(6.7)$ & $5.7(6.9)$ & $3.7(8.0)$ & $2.6(8.9)$ \\
social function & $5.8(10.7)$ & $7.8(7.5)$ & $-1.3(8.1)$ & $6.3(9.0)$ \\
role emotional & $5.1(12.6)$ & $9.0(16.2)$ & $3.3(13.1)$ & $2.8(6.6)$ \\
mental health & $2.0(7.0)$ & $4.9(6.6)$ & $2.0(5.9)$ & $4.9(9.7)$ \\
PCS & $7.9(7.0)$ & $5.9(7.4)$ & $6.9(9.7)$ & $2.3(6.6)$ \\
MCS & $1.4(10.1)$ & $6.2(8.4)$ & $-0.3(6.7)$ & $17.0(39.7)$ \\
Total FES score & $9.3(32.3)$ & $8.2(32.7)$ & $7.5(45.6)$ & \\
\hline Ipsilayyy
\end{tabular}

Ipsilateral, ipsilateral side; Contra, contralateral side

${ }^{*} \mathrm{p}<0.05$ : a significant difference, Mann-Whitney's U test. SD: standard deviation, FES: fall efficacy scale; FIM: functional independence measure; GDS-15: geriatric depression scale short form (15 items); MCS: mental component summary; MMSE: mini-mental state examination; PCS: physical component summary; SF-8: 8-item short-form health survey; TUG: timed up and go test

mental (effect size shown in Table 2). In addition, grip strength and MMSE were maintained, and there were no negative effects on general condition or cognitive function during the hospitalization period of about 1 month. Ambulatory status with use of an assistive device in the TKA and THA groups at admission showed that $10(18 \%)$ and $1(2 \%)$ had independent ambulation, $36(64 \%)$ and $26(67 \%)$ used cane-assisted ambulation, and $10(18 \%)$ and $12(31 \%)$ used walker-assisted ambulation, respectively, while those values at discharge were $27(48 \%)$ and $4(10 \%), 29(52 \%)$ and $33(85 \%)$, and $0(0 \%)$ and 2 $(10 \%)$, respectively.

Using 65 years old as the cut-off, the effects of rehabilitation were examined with a delta score by subtracting the findings at admission from those at discharge. Patients in the THA group who were $\geq 65$ years of age had worse improvements in QOL with regard to bodily pain and self-efficacy in avoiding falls, whereas there were no significant differences in the TKA group (Table 3). When grouped on the basis of 29 points as the median MMSE score for cognitive function, patients in the TKA group with a score of $\leq 28$ showed a worse effect of rehabilitation on knee extension strength on the left side (many patients had surgery on the non-dominant leg) (Table 4), while those in the THA group with a low score had worse improvement in the FES score (Table 5). When grouped on the basis of the number of comorbidities, patients with 2 or more in the THA group showed a significantly lower rehabilitation effect of vitality on HRQOL and FES scores, while there was no significant difference in the TKA group (Table 6).

\section{DISCUSSION}

In this study, changes in motor function, cognitive function, mood, and HRQOL were assessed in patients who underwent a TKA or THA procedure at 2 and 4 weeks after surgery (non-acute stage), and the CGA was used to determine the effects of convalescence rehabilitation. Scores for independence in ADL (FIM sub-items $\geq 5$ points) from the time of admission showed that both motor function and HRQOL significantly improved in both groups. Therefore, convalescence rehabilitation for TKA and THA patients is effective for a wide range of factors, and CGA was found to be useful even for patients with lower extremity joint replacement. Moreover, the clinical significance of various measures resulting from extension of the short-term PT intervention was confirmed, indicating that extension of the hospitalization period due to rehabilitation medi- 
Table 5. Differences between admission and discharge based on cognitive function

\begin{tabular}{lcccc}
\hline \multirow{2}{*}{$\Delta)=$ Discharge - Admission } & \multicolumn{2}{c}{ Total knee, mean $(\mathrm{SD})$} & \multicolumn{2}{c}{ Total hip, mean (SD) } \\
\cline { 2 - 5 } MMSE $^{\dagger}$ & $\begin{array}{c}\text { High, } \geq 29 \\
(\mathrm{n}=34)\end{array}$ & $\begin{array}{c}\text { Low, }<29 \\
(\mathrm{n}=22)\end{array}$ & $\begin{array}{c}\text { High, } \geq 29 \\
(\mathrm{n}=24)\end{array}$ & $\begin{array}{c}\text { Low, }<29 \\
(\mathrm{n}=15)\end{array}$ \\
\hline Total FIM score, capability & $7.4(4.5)$ & $8.3(4.3)$ & $7.5(3.1)$ & $9.3(4.8)$ \\
FIM efficiency & $0.5(0.3)$ & $0.5(0.2)$ & $0.5(0.2)$ & $0.5(0.3)$ \\
Grip, right $(\mathrm{kg})$ & $0.2(2.5)$ & $-0.7(2.5)$ & $0.2(2.4)$ & $0.4(2.8)$ \\
Grip, left $(\mathrm{kg})$ & $0.4(2.2)$ & $0.2(2.4)$ & $0.4(2.7)$ & $0.1(1.7)$ \\
Knee extension, right $(\mathrm{Nm} / \mathrm{kg})$ & $0.2(0.2)$ & $0.2(0.2)$ & $0.1(0.2)$ & $0.2(0.1)$ \\
Knee extension, left $(\mathrm{Nm} / \mathrm{kg})$ & $0.2(0.2)$ & $0.1(0.2)^{*}$ & $0.1(0.2)$ & $0.2(0.1)$ \\
TUG (sec) & $-3.7(4.3)$ & $-3.7(3.3)$ & $-3.9(4.1)$ & $-4.0(4.6)$ \\
Total MMSE score & $-0.3(1.1)$ & $0.2(2.2)$ & $-0.3(1.3)$ & $0.5(1.9)$ \\
Total GDS-15 score & $-1.2(2.2)$ & $-2.0(2.8)$ & $-1.0(1.7)$ & $0.5(2.8)$ \\
SF-8 score & & & & \\
physical function & $4.9(9.4)$ & $11.0(11.1)^{*}$ & $4.4(9.8)$ & $6.4(13.2)$ \\
role physical & $8.6(12.7)$ & $7.9(10.7)$ & $6.4(10.0)$ & $5.4(13.6)$ \\
bodily pain & $5.1(7.6)$ & $5.2(6.0)$ & $6.9(8.8)$ & $3.3(9.8)$ \\
general health & $3.8(7.8)$ & $4.8(8.8)$ & $5.2(8.3)$ & $0.3(4.8)$ \\
vitality & $5.1(6.8)$ & $2.2(6.8)$ & $3.0(7.1)$ & $1.2(7.2)$ \\
social function & $7.0(10.2)$ & $6.2(8.3)$ & $1.2(8.1)$ & $-1.3(10.0)$ \\
role emotional & $6.6(14.6)$ & $6.9(14.1)$ & $6.6(11.6)$ & $-0.3(9.3)$ \\
mental health & $3.8(7.2)$ & $2.3(6.4)$ & $1.6(6.3)$ & $4.3(5.6)$ \\
PCS & $6.0(7.0)$ & $8.7(7.2)$ & $6.5(8.9)$ & $4,7(11.6)$ \\
MCS & $4.5(10.2)$ & $1.9(8.8)$ & $1.4(7.1)$ & $-0.5(5.7)$ \\
Total FES score & $10.1(29.2)$ & $7.0(37.1)$ & $24.9(32.0)$ & $-21.1(49.9)^{*}$ \\
\hline
\end{tabular}

${ }^{\dagger}$ The high- and low-scoring groups (High, Low) were distinguished using the median MMSE score (29 points for both TKA and THA groups).

${ }^{*} \mathrm{p}<0.05$ : a significant difference, Mann-Whitney's U test. SD: standard deviation; FES: fall efficacy scale; FIM: functional independence measure; GDS-15: geriatric depression scale short form (15 items); MCS: mental component summary; MMSE: mini-mental state examination; PCS: physical component summary; SF-8: 8-item short-form health survey; TUG: timed up and go test

cal treatment for about 2 weeks after ADL independence is effective.

Recently, there has been a trend in Japan to reduce the length of hospital stay. However, based on the present results, it is our opinion that discharge home after rehabilitation therapy with hospitalization within 2 weeks is insufficient, especially for elderly patients, while 4 weeks or more is beneficial. In Japanese cases, CRW plays a role as a bridge to community rehabilitation. In the present study, the subjects were divided into 2 groups based on a cut-off of 65 years old, which is the age of eligibility for receiving long-term care insurance. This study found that patients $\geq 65$ years of age who underwent THA had poor improvement in HRQOL with respect to pain and self-efficacy for preventing falls. In contrast, in the TKA group, there was no significant difference seen after that age division; however, there was only a small number of patients under 65 years old. Also, a comparison of cognitive function using the median MMSE score showed that improvement of knee extension strength in the TKA group, and FES in the THA group, were also poor. Similarly, numerous co-morbidities were related to poor improvement in FES in the THA group. Therefore, development of a more effective PT program for geriatric individuals undergoing TKA or THA is an important issue to address in the future. In addition, there are concerns regarding housebound individuals and locomotive syndrome after discharge, as there are specific problems of elderly patients. It has been reported that elderly patients who underwent TKA and received PT for less than 1 month showed decreased gait ability and muscle activity compared to healthy elderly subects ${ }^{16)}$. Thus, PT intervention for TKA and THA patients for both body and mind, to improve ADL after discharge, is important until they reach a high level of physical activity.

In our study, improvements in depressive mood and locomotive function over a short period were observed, and the amount of activity other than PT is expected to rapidly increase in post-operative patients. If patients are discharged after achieving full functional improvement, a higher level of physical activity is also assured, indicating that ADL/QOL and healthy life expectancy will continue on a natural course. In previous studies of physical activity and clinical outcomes, as opposed to pre-operative findings ${ }^{17}$ ), post-operative findings were reported to correlate with functional improvements and amount of walking shortly after surgery ${ }^{18-20)}$ in patients who underwent a TKA or THA procedure. Thus, improvement to achieve sufficiency gait function during hospitalization is important. In cases of early hospital discharge, it is also important 
Table 6. Differences in functional improvement based on number of comorbidities

\begin{tabular}{lcccc}
\hline \multirow{2}{*}{$\Delta)=$ Discharge - Admission } & \multicolumn{2}{c}{ Total knee, mean $(\mathrm{SD})$} & \multicolumn{2}{c}{ Total hip, mean (SD) } \\
\cline { 2 - 4 } Comorbidities & 0 or 1 & $\geq 2$ & 0 or 1 & $\geq 2$ \\
& $(\mathrm{n}=24)$ & $(\mathrm{n}=32)$ & $(\mathrm{n}=25)$ & $(\mathrm{n}=14)$ \\
\hline Total FIM score, capability & $7.0(4.7)$ & $8.3(4.2)$ & $7.7(3.2)$ & $8.6(4.5)$ \\
FIM efficiency & $0.5(0.3)$ & $0.5(0.3)$ & $0.5(0.2)$ & $0.5(0.2)$ \\
Grip, right $(\mathrm{kg})$ & $-0.4(2.4)$ & $0.0(2.6)$ & $-0.2(2.7)$ & $0.9(2.0)$ \\
Grip, left $(\mathrm{kg})$ & $0.2(2.6)$ & $0.3(2.0)$ & $0.5(2.7)$ & $-0.1(2.0)$ \\
Knee extension, right $(\mathrm{Nm} / \mathrm{kg})$ & $0.2(0.3)$ & $0.2(0.2)$ & $0.1(0.2)$ & $0.1(0.2)$ \\
Knee extension, left $(\mathrm{Nm} / \mathrm{kg})$ & $0.2(0.2)$ & $0.1(2.0)$ & $0.1(0.2)$ & $0.2(0.2)$ \\
TUG (sec) & $-3.1(3.4)$ & $-4.2(4.3)$ & $-3.8(3.8)$ & $-4.3(4.8)$ \\
Total MMSE score & $0.3(1.7)$ & $-0.4(1.5)$ & $-0.2(1.5)$ & $0.1(1.7)$ \\
Total GDS-15 score & $-1.5(2.5)$ & $-1.6(2.5)$ & $-0.8(1.6)$ & $-0.1(2.9)$ \\
SF-8 score & & & & $5.0(8.6)$ \\
physical function & $8.5(11.5)$ & $6.4(9.7)$ & $4.9(12.0)$ & $4.6(12.1)$ \\
role physical & $7.3(12.2)$ & $9.1(11.7)$ & $7.0(10.5)$ & $3.7(9.5)$ \\
bodily pain & $5.2(7.6)$ & $5.1(6.6)$ & $7.1(8.8)$ & $2.0(8.3)$ \\
general health & $3.5(8.9)$ & $4.7(7.6)$ & $4.9(7.3)$ & $-1.2(6.3)^{*}$ \\
vitality & $2.7(7.4)$ & $4.9(6.4)$ & $4.5(6.7)$ & $-0.9(9.3)$ \\
social function & $6.9(10.5)$ & $6.5(8.7)$ & $1.3(8.3)$ & $5.2(6.2)$ \\
role emotional & $6.3(15.3)$ & $7.1(13.6)$ & $4.4(13.5)$ & $3.0(8.6)$ \\
mental health & $4.0(5.6)$ & $2.6(7.8)$ & $2.0(4.4)$ & $3.7(9.1)$ \\
PCS & $6.7(7.3)$ & $7.3(7.2)$ & $7.2(9.9)$ & $1.1(6.1)$ \\
MCS & $3.5(9.6)$ & $3.5(9.8)$ & $0.8(7.1)$ & $-5.3(57.5)^{*}$ \\
Total FES score & $4.8(32.1)$ & $11.9(32.5)$ & $21.5(28.8)$ & \\
\hline
\end{tabular}

${ }^{*} \mathrm{p}<0.05$ : a significant difference, Mann-Whitney's U test. SD: standard deviation; FES: fall efficacy scale; FIM: functional independence measure; GDS-15: geriatric depression scale short form (15 items); MCS: mental component summary; MMSE: mini-mental state examination; PCS: physical component summary; SF-8: 8-item short-form health survey; TUG: timed up and go test

for physical therapists to advise patients to find opportunities to walk and perform a rehabilitation program at home, without having to continue with aggressive $\mathrm{PT}^{21)}$.

This study had several limitations, including the lack of a control group and no multicentric characteristics. Future largescale studies would be helpful to establish the usefulness of post-operative rehabilitation for TKA and THA patients in CRW. Despite these limitations, the present findings indicate that patients who undergo TKA or THA should show improved motor function and HRQOL during the very early weeks of hospitalization. Although we suggest that the hospitalization period be commensurate with the aging society in Japan, for younger working patients, a fast-track course is recommended. Compared to UDSMR-FIM, the ADL scores of our patients were high; thus, it will be necessary to conduct further analyses with longterm follow-up as well as assessments of cost-effectiveness after discharge. Visser et al. ${ }^{22)}$ reported that functional ability was maintained and showed an improving trend at 6 months after surgery, and the level of functional ability was maintained at approximately 4 years later in patients who underwent TKA or THA, while their amounts of physical activity did not increase. Brandes et al. ${ }^{3)}$ also reported that physical activity parameters did not correlate with clinical outcomes during the first year after TKA, while Nilsdotter et al. found that the peak of physical functioning was observed at 1 year after surgery, and then declined thereafter up to 5 years post-TKA and 7 years post-THA ${ }^{23,24)}$. In a future study, it will be necessary to verify the impact of a hospitalization period extension of about 2 weeks on medium- and long-term clinical results.

Cognitive decline in the elderly after a TKA or THA procedure is a concern. A previous study reported slightly reduced cognitive function at discharge compared to before surgery in total joint arthroplasty (TJA) patients, though evidence of decline for the period at 3-6 months after surgery was not clear ${ }^{25}$. Although our study included a large percentage of elderly individuals, we found no cognitive decline during hospitalization, as shown by MMSE scores. It is known that exercise has a positive impact on both movement and cognitive function ${ }^{26)}$, and is a factor related to mental health ${ }^{27)}$; thus, our patients might have gained motor and cognitive function improvements through PT. Also, though the number of falls during hospitalization in our cohort was small, a relationship between hip fracture caused by falls and cataracts in elderly individuals has been shown ${ }^{28)}$; thus, patient guidance that takes into account falls in advance of surgery is important. In addition, it is not uncommon for postoperative delirium to occur in elderly patients after a TKA or THA procedure ${ }^{29-31)}$. Therefore, routine screening for postoperative delirium and cognitive impairment using the MMSE or another device is important for elderly cases. 


\section{ACKNOWLEDGEMENTS}

We express their appreciation to all who provided support until the closure of the convalescence rehabilitation ward of our hospital in April 2014. None of the authors has any conflicts of interest or financial ties to disclose. This study was approved by the research ethics committee of Shiga University of Medical Science (24-3).

\section{REFERENCES}

1) Westby MD, Brittain A, Backman CL: Expert consensus on best practices for post-acute rehabilitation after total hip and knee arthroplasty: a Canada and United States Delphi study. Arthritis Care Res (Hoboken), 2014, 66: 411-423. [Medline] [CrossRef]

2) Peter WF, Dekker J, Tilbury C, et al.: The association between comorbidities and pain, physical function and quality of life following hip and knee arthroplasty. Rheumatol Int, 2015, 35: 1233-1241. [Medline] [CrossRef]

3) Brandes M, Ringling M, Winter C, et al.: Changes in physical activity and health-related quality of life during the first year after total knee arthroplasty. Arthritis Care Res (Hoboken), 2011, 63: 328-334. [Medline]

4) Ethgen $\mathrm{O}$, Bruyère $\mathrm{O}$, Richy $\mathrm{F}$, et al.: Health-related quality of life in total hip and total knee arthroplasty. A qualitative and systematic review of the literature. J Bone Joint Surg Am, 2004, 86-A: 963-974. [Medline]

5) Jones CA, Pohar S: Health-related quality of life after total joint arthroplasty: a scoping review. Clin Geriatr Med, 2012, 28: 395-429. [Medline] [CrossRef]

6) Daigle ME, Weinstein AM, Katz JN, et al.: The cost-effectiveness of total joint arthroplasty: a systematic review of published literature. Best Pract Res Clin Rheumatol, 2012, 26: 649-658. [Medline] [CrossRef]

7) Granger CV, Markello SJ, Graham JE, et al.: The uniform data system for medical rehabilitation: report of patients with lower limb joint replacement discharged from rehabilitation programs in 2000-2007. Am J Phys Med Rehabil, 2010, 89: 781-794. [Medline] [CrossRef]

8) Westby MD: Rehabilitation and total joint arthroplasty. Clin Geriatr Med, 2012, 28: 489-508. [Medline] [CrossRef]

9) Rat AC, Guillemin F, Osnowycz G, et al.: Total hip or knee replacement for osteoarthritis: mid- and long-term quality of life. Arthritis Care Res (Hoboken), 2010, 62: 54-62. [Medline] [CrossRef]

10) Ellis G, Whitehead MA, O’Neill D, et al.: Comprehensive geriatric assessment for older adults admitted to hospital. Cochrane Database Syst Rev, 2011, (7): CD006211. [Medline]

11) Scanlan BC: The value of comprehensive geriatric assessment. Care Manag J, 2005, 6: 2-8. [Medline] [CrossRef]

12) Stuck AE, Siu AL, Wieland GD, et al.: Comprehensive geriatric assessment: a meta-analysis of controlled trials. Lancet, 1993, 342: 1032-1036. [Medline] [CrossRef]

13) Takenaka K, Chikagawa M, Honda G, et al.: Development of falling self-efficacy scale for elderly people: the reliability and validity. Taiikugaku kenkyu (Japan Journal of Physical Education, Health and Sport Sciences), 2002, 47 : 1-13 (in Japanese).

14) Oldfield RC: The assessment and analysis of handedness: the Edinburgh inventory. Neuropsychologia, 1971, 9: 97-113. [Medline] [CrossRef]

15) Elias LJ, Bryden MP, Bulman-Fleming MB: Footedness is a better predictor than is handedness of emotional lateralization. Neuropsychologia, 1998, 36: 37-43. [Medline] [CrossRef]

16) Lee A, Park J, Lee S: Gait analysis of elderly women after total knee arthroplasty. J Phys Ther Sci, 2015, 27: 591-595. [Medline] [CrossRef]

17) Poortinga S, van den Akker-Scheek I, Bulstra SK, et al.: Preoperative physical activity level has no relationship to the degree of recovery one year after primary total hip or knee arthroplasty: a cohort study. PLoS ONE, 2014, 9: e115559. [Medline] [CrossRef]

18) Lützner C, Kirschner S, Lützner J: Patient activity after TKA depends on patient-specific parameters. Clin Orthop Relat Res, 2014, 472: 3933-3940. [Medline] [CrossRef]

19) Ko V, Naylor JM, Harris IA, et al.: The six-minute walk test is an excellent predictor of functional ambulation after total knee arthroplasty. BMC Musculoskelet Disord, 2013, 14: 145. [Medline] [CrossRef]

20) Kuhn M, Harris-Hayes M, Steger-May K, et al.: Total hip arthroplasty in patients 50 years or less: do we improve activity profiles? J Arthroplasty, 2013, 28: 872-876. [Medline] [CrossRef] 
21) Büker N, Akkaya S, Akkaya N, et al.: Comparison of effects of supervised physiotherapy and a standardized home program on functional status in patients with total knee arthroplasty: a prospective study. J Phys Ther Sci, 2014, 26 : 1531-1536. [Medline] [CrossRef]

22) Vissers MM, Bussmann JB, de Groot IB, et al.: Physical functioning four years after total hip and knee arthroplasty. Gait Posture, 2013, 38: 310-315. [Medline] [CrossRef]

23) Nilsdotter AK, Toksvig-Larsen S, Roos EM: A 5 year prospective study of patient-relevant outcomes after total knee replacement. Osteoarthritis Cartilage, 2009, 17: 601-606. [Medline] [CrossRef]

24) Nilsdotter AK, Isaksson F: Patient relevant outcome 7 years after total hip replacement for OA—a prospective study. BMC Musculoskelet Disord, 2010, 11: 47. [Medline] [CrossRef]

25) Scott JE, Mathias JL, Kneebone AC: Postoperative cognitive dysfunction after total joint arthroplasty in the elderly: a meta-analysis. J Arthroplasty, 2014, 29: 261-7.e1. [Medline] [CrossRef]

26) Angevaren M, Aufdemkampe G, Verhaar HJ, et al.: Physical activity and enhanced fitness to improve cognitive function in older people without known cognitive impairment. Cochrane Database Syst Rev, 2008, (3): CD005381. [Medline]

27) Mattson MP: Energy intake and exercise as determinants of brain health and vulnerability to injury and disease. Cell Metab, 2012, 16: 706-722. [Medline] [CrossRef]

28) Tseng VL, Yu F, Lum F, et al.: Risk of fractures following cataract surgery in Medicare beneficiaries. JAMA, 2012, 308: 493-501. [Medline] [CrossRef]

29) Rade MC, Yadeau JT, Ford C, et al.: Postoperative delirium in elderly patients after elective hip or knee arthroplasty performed under regional anesthesia. HSS J, 2011, 7: 151-156. [Medline] [CrossRef]

30) Bin Abd Razak HR, Yung WY: Postoperative delirium in patients undergoing total joint arthroplasty: a systematic review. J Arthroplasty, 2015, 30: 1414-1417. [Medline] [CrossRef]

31) Zywiel MG, Prabhu A, Perruccio AV, et al.: The influence of anesthesia and pain management on cognitive dysfunction after joint arthroplasty: a systematic review. Clin Orthop Relat Res, 2014, 472: 1453-1466. [Medline] [CrossRef] 